Raúl Zarca, Alfredo Ortiz*, Daniel Gorri and Inmaculada Ortiz

\title{
Facilitated Transport of Propylene Through Composite Polymer-lonic Liquid Membranes. Mass Transfer Analysis
}

DOI 10.1515/cppm-2015-0072

Received December 22, 2015; accepted December 22, 2015

\begin{abstract}
Separation of light gaseous olefins from paraffin's of the refinery process off-gasses has been traditionally performed by cryogenic distillation, which is a highly capital and energy intensive operation. This handicap creates an incentive for the investigation of alternative olefin/paraffin separation technologies. In this regard, membrane technology supposes a potential solution for process intensification. Previous works of our research group reported the use of facilitated transport composite membranes integrating the use of PVDF-HFP polymer, $\mathrm{BMImBF}_{4}$ ionic liquid and $\mathrm{AgBF}_{4}$ silver salt. In this type of membranes, the silver cations react selectively and reversibly with the olefin, allowing the separation via mobile and fixed carrier mechanisms. Ionic liquids were selected as membrane additives because in addition to their negligible vapor pressure that avoids solvent losses by evaporation, they provide stability to the metallic cation dissolved inside, and modify the structure improving the facilitated transport. This technology offers a commercial attractive separation alternative thanks to their modular form of operation, high values of selectivity and permeability and low operational costs. In the present work, propane/propylene permeation experiments involving the use ionic liquids and different membrane compositions were performed. Moreover, basing on the transport and equilibrium parameters previously obtained, a mathematical model description of the system will be proposed fitting the remaining parameters and allowing the design and optimization of the propane/propylene separation process at industrial levels.
\end{abstract}

Keywords: facilitated transport, propylene, ionic liquids, silver, membrane composite

\footnotetext{
*Corresponding author: Alfredo Ortiz, Department of Chemical \& Biomolecular Engineering, University of Cantabria, Av. Los Castros s/n., 39005 Santander, Spain, E-mail: alfredo.ortizsainz@unican.es Raúl Zarca, Daniel Gorri, Inmaculada Ortiz, Department of Chemical \& Biomolecular Engineering, University of Cantabria, Av. Los Castros s/n., 39005 Santander, Spain
}

\section{Introduction}

Light olefins such as ethylene and propylene are important petrochemical building blocks which are further processed to yield a wide range of final products such as cosmetics, textile products, paints, tools or plastics for instance. Light olefins are usually obtained, as a mixture with paraffins, by steam cracking processes, fluidized catalytic cracking or alkane dehydrogenation.

The separation of these streams is a key issue because it is one of the most difficult and also the most costly separation process in the petrochemical industry. Traditional separation processes like low-temperature distillation, require voluminous equipment operating at high pressures or low temperatures and very large reflux ratios due to the very small difference in the relative volatilities between olefins and their corresponding paraffins [1]. In recognition of these costs alternative energysaving separation processes are required.

In this sense, membrane technology presents a great potential for energy and capital saving and therefore the use of membranes has been the focal point of research of many authors. Criteria for selecting the most suitable membrane for a given application are complex; nonetheless, durability, mechanical and thermal stability at the operating conditions, productivity and separation efficiency and costs are important stipulations that must be considered [2]. Among these requirements selectivity and permeation rate are clearly the most basic, while the relative importance of the rest of them varies with the application. Therefore a wide variety of different membrane alternatives can be considered [3]. The use of polymeric, inorganic and supported liquid membranes for olefin/ paraffin separation has been studied rather extensively, however these membranes may suffer severe plasticization effect, low stability, poor mechanical resistance and expensive and complex preparation methods and therefore their performance has not met the requirements for commercial applications $[4,5]$.

Recently our research group proposed the use of novel polymer-ionic liquid- $\mathrm{Ag}^{+}$composite as facilitated transport membranes to carry out the separation of 
propane/propylene mixtures [6]. The separation performance is mostly associated to the ability of the olefins to react selective and reversibly with silver cations $\mathrm{Ag}^{+}$, by $\pi$-complexation mechanism. Consequently silver ions act as carriers for the transport of olefins, thus facilitating their selective permeation across the membrane $[7,8]$. Ionic liquids (IL) were selected as membrane additives because in addition to their negligible vapor pressure that avoids solvent losses by evaporation, they offer more affinity for the olefinic compounds compared to saturated hydrocarbons and at the same time they provide stability to the metallic cation dissolved inside acting as a medium for facilitated transport with mobile carrier $[9,10]$. Based on previous results the ionic liquid used in this work has been 1-butyl 3-methylimidazolium tetrafluoroborate $\left(\mathrm{BMImBF}_{4}\right)$ since it provided the best results in terms of separation selectivity and propylene solubility [11-13]. On the other hand, the polymer used in this work is poly(vinylidene fluoride-co-hexafluoropropylene) (PVDF-HFP) due to its well-known thermal, chemical and mechanical properties. Furthermore it is partially miscible with $\mathrm{BMImBF}_{4}$ and compatible with hydrocarbons which avoid plasticization effects. Once the membrane is fabricated the ionic liquid remains integrated within the polymer matrix, thereby increasing the stability of the membrane even at high transmembrane pressures. As the composite membrane is composed by a polymeric matrix and a liquid phase entrapped inside the polymeric matrix, the silver salt added to the membrane is distributed between these two phases. Therefore both facilitated transport mechanisms (fixed carrier and mobile carrier) take place, leading to high permeabilities combined with high separation selectivities. Figure 1 shows and schematic diagram of the transport mechanisms of propane and propylene across polymer/ionic liquid composite membrane. The PVDF-HFP/BMImBF $4^{-}$ $\mathrm{Ag}^{+}$facilitated transport membranes reported in a previous work provided very promising results when tested

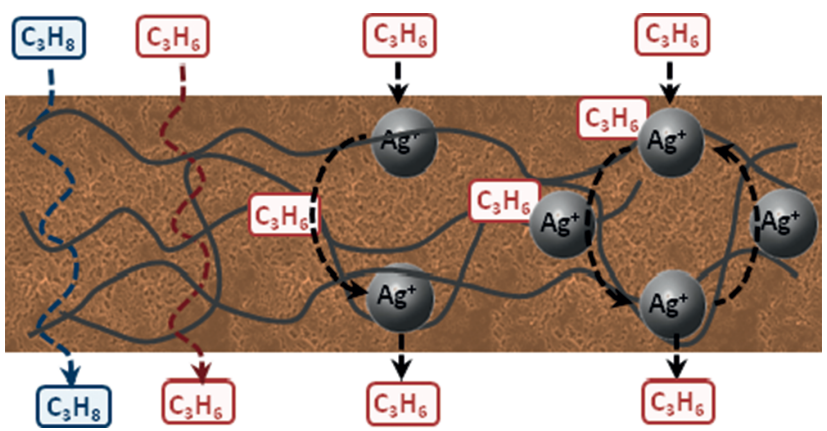

Figure 1: Proposed facilitated transport composite membrane structure. with $50 / 50 \%$ v/v $\mathrm{C}_{3} \mathrm{H}_{8} / \mathrm{C}_{3} \mathrm{H}_{6}$ mixtures obtaining $\mathrm{C}_{3} \mathrm{H}_{6}$ permeabilities up to 6,630 barrer and $\mathrm{C}_{3} \mathrm{H}_{8} / \mathrm{C}_{3} \mathrm{H}_{6}$ selectivities over 700 combined with good long term stability [6].

In this work permeation experiments using constant volume time-lag method were performed in order to obtain diffusivity and solubility parameters of propylene and propane in PVDF-HFP polymer matrix and ionic liquid/polymer composite membranes. These results combined with facilitated transport experimental data obtained from our previous works $[6,11]$ will allow the development of a mathematical model able to describe the behavior of the facilitated transport mechanisms of propylene through the composite membrane and estimate the unknown facilitated transport parameters.

\section{Experimental section}

\subsection{Membrane preparation}

The PVDF-HFP/BMImBF 4 composite membranes were prepared by the solvent casting technique. First of all the polymer is dissolved in $10 \mathrm{~mL}$ of THF at $40^{\circ} \mathrm{C}$ for $8 \mathrm{~h}$ in a closed vial to avoid the evaporation of the solvent. Once the polymer has been dissolved, it is mixed with the ionic liquid and stirred for $5 \mathrm{~min}$. Finally the membrane precursor mixture is placed in a Petri dish and the solvent is evaporated at $25^{\circ} \mathrm{C}$ and 300 mbar under dark conditions for $24 \mathrm{~h}$. The thickness of the membranes prepared in this work depends on the membrane composition, but in all cases the prepared membranes presented a range thickness between 40-100 $\mu \mathrm{m}$. Permeabilities have been calculated taking into account the real thickness of each membrane which was measured using a digital micrometer Mitutoyo Digmatic Series 369 (accuracy $\pm 0.001 \mathrm{~mm}$ ).

\subsection{Time-lag experiments}

Figure 2 shows the experimental setup used for conducting permeation experiments using the time-lag technique at constant-volume and variable pressure. This apparatus consists of two chambers separate by the membrane ( $47 \mathrm{~mm}$ diameter). At time zero the gas of interest is introduced into the upper chamber. The feed pressure is maintained constant at the upper chamber while the pressure increase in the lower chamber, which occurs due to the passage of gas through the membrane, is recorded. The appreciable increase in pressure in the 


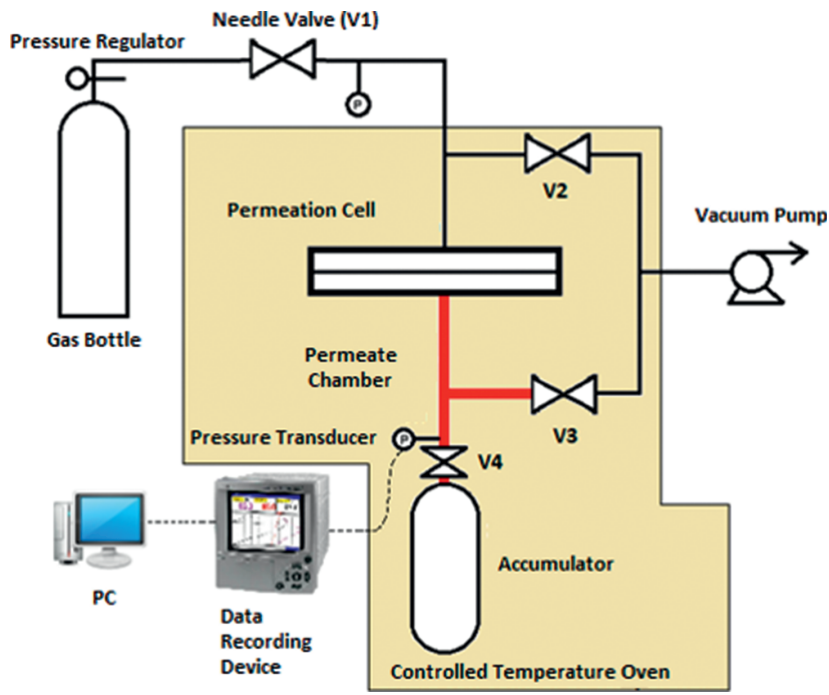

Figure 2: Time-lag apparatus scheme.

lower chamber occurs only after a period of time known as time-lag $(\theta)$. After the time-lag a diffusion process begins in quasi-steady state until the pressure in both chambers is equalized. The mathematical expression that describes the increased pressure in the lower chamber can be obtained by applying Fick's second law in the limits of the membrane. By integrating Fick's equation with the boundary conditions by Laplace transforms, operating and neglecting terms, we reach the expression for the pressure in the permeate chamber versus time for the quasi-steady flux:

$$
P_{L}(t)=A \frac{R \cdot T \cdot S \cdot D \cdot P_{0}}{V \cdot L}\left(t-\frac{L^{2}}{6 D}\right)
$$

The above equation is a straight line, from which one can extract the term that subtracts the time, known as "timelag” $(\theta)$ and the slope:

$$
\theta=\frac{L^{2}}{6 D}
$$

After re-arranging terms, diffusivity, solubility and permeability parameters can be obtained as:

$$
\begin{gathered}
D=\frac{L^{2}}{6 \theta} \\
S=\frac{V \cdot L \cdot(\text { Slope })}{A \cdot D \cdot R \cdot T \cdot P_{0}} \\
P=D \cdot S
\end{gathered}
$$

Where, $\mathrm{L}$ is the membrane thickness, $\theta$ is the "time-lag", $\mathrm{V}$ is the permeate side volume, $\mathrm{A}$ is the permeation area, $R$ is the gas constant, $T$ is temperature and $P_{0}$ is the feed pressure.

\section{Results and discussion}

The results of permeation experiments are discussed. Permeability of propylene, propane and carbon dioxide, as a reference gas, at different temperatures in PVDF-HFP and PVDF-HFP-BMImB 4 (80/20\%wt.) membranes are experimentally obtained.

Figure 3 shows the propylene permeation time lag experiment at $289 \mathrm{~K}$ in a PVDF-HFP membrane. The characteristic initial stage corresponds to the time-lag $(\theta)$ and the straight line corresponds to the quasi-steady state permeation stage. We can observe the long duration required for the experiment to reach quasi-steady state permeation flux and the slight increase in pressure in the permeate chamber, which gives an idea of the low permeability values expected in this polymer. In all cases the supply pressure described here is 3.5 bar. The permeate chamber has a volume of $16.6 \mathrm{~cm}^{3}$.

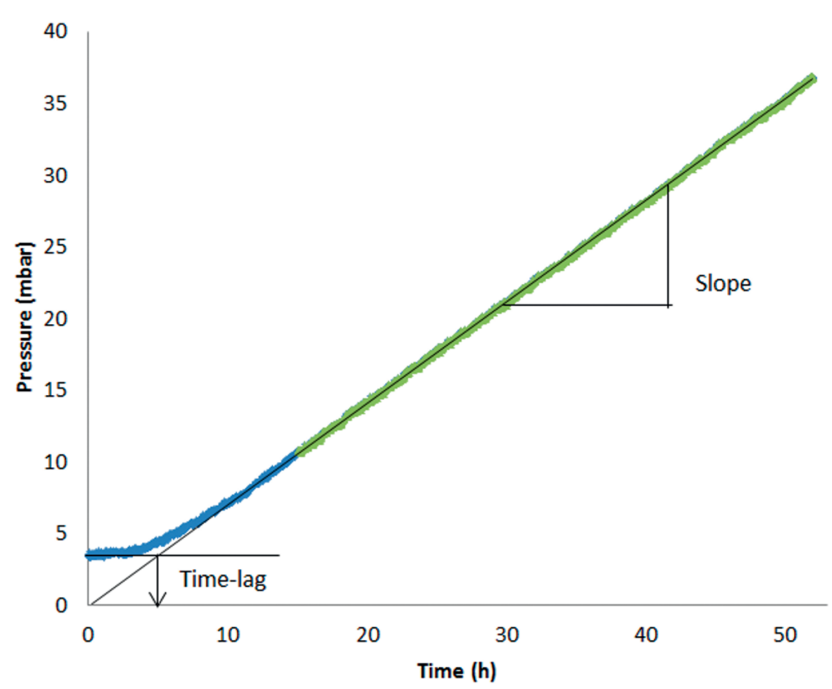

Figure 3: Permeate side pressure increase for propylene in PVDF-HFP membrane at $298 \mathrm{~K}$.

Table 1 summarizes the experimental values of diffusivity, solubility and permeability of $\mathrm{CO}_{2}$, propane and propylene in PVDF-HFP membranes at different temperatures.

Permeability of $\mathrm{CO}_{2}$ in the dense polymer membrane is an order of magnitude higher than that of propylene and propane. This effect is due to a significant increase in diffusivity because the smaller size of the $\mathrm{CO}_{2}$ molecule.

Furthermore, permeability values for propylene and propane are very similar. So it is confirmed that this type of dense membranes, in which the mass transfer takes place only by the solution-diffusion mechanism, cannot be used to carry out the separation of propane/propylene 
Table 1: Diffusivity, solubility and permeability of $\mathrm{CO}_{2}$, propane and propylene PVDF-HFP 298, 308 and 318 K.

\begin{tabular}{|c|c|c|c|c|}
\hline & Temperature & D & $\mathbf{s}$ & $\mathbf{P}$ \\
\hline & (K) & $\left(\times 10^{9} \mathrm{~cm}^{2} / \mathrm{s}\right)$ & $\left(\times 10^{5} \mathrm{~mol} / \mathrm{bar} / \mathrm{cm}^{3}\right)$ & (barrer) \\
\hline \multirow[t]{3}{*}{$\mathrm{CO}_{2}$} & 298 & 2.16 & 6.12 & 0.373 \\
\hline & 308 & 4.44 & 5.79 & 0.811 \\
\hline & 318 & 11.6 & 4.12 & 1.430 \\
\hline \multirow[t]{3}{*}{ Propylene } & 298 & 0.13 & 9.24 & 0.035 \\
\hline & 308 & 0.4 & 6.31 & 0.075 \\
\hline & 318 & 1.97 & 2.84 & 0.167 \\
\hline \multirow[t]{3}{*}{ Propane } & 298 & 0.04 & 7.78 & 0.010 \\
\hline & 308 & 0.11 & 11.2 & 0.036 \\
\hline & 318 & 0.16 & 11.8 & 0.058 \\
\hline
\end{tabular}

Table 2: Diffusivity of $\mathrm{CO}_{2}$, propane and propylene in PVDF-HFP at $298 \mathrm{~K}$ and Lennard-Jones diameter [14].

\begin{tabular}{lrr}
\hline & $\mathbf{D}\left(\times \mathbf{1 0} \mathbf{c m}^{\mathbf{2}} \mathbf{s}\right)$ & Lennard-Jones diameter $(\AA)$ \\
\hline $\mathrm{CO}_{2}$ & 2.16 & 3.94 \\
Propylene & 0.13 & 4.68 \\
Propane & 0.04 & 5.12 \\
\hline
\end{tabular}

mixtures. For the three pure gases is observed that the diffusion coefficients are inversely proportional to their kinetic diameters, as shown in Table 2.

Results in Table 1 show a significant increase in permeability when temperature increases. The temperature significantly increases diffusivity whereas the solubility decreases slightly. Activation energies for the three gases using Arrhenius equation were calculated in order to know the influence of temperature on the permeation process. Activation energies are shown in Table 3, from which it can be observed that the permeation of propane is more sensitive to temperature than propylene and being the lowest for the $\mathrm{CO}_{2}$.

Table 3: Activation energies of $\mathrm{CO}_{2}$, propylene and propane in PVDF-HFP.

\begin{tabular}{lr}
\hline Gas & $\mathbf{E}_{\mathbf{a}}(\mathbf{k J} / \mathbf{m o l})$ \\
\hline $\mathrm{CO}_{2}$ & 53 \\
Propylene & 61 \\
Propane & 69 \\
\hline
\end{tabular}

In order to study the influence of the addition of an ionic liquid to the polymer matrix permeation experiments in an
$80 \%$ PVDF-HFP and $20 \%$ of $\mathrm{BMImBF}_{4}$ composite membrane were conducted. The addition of the ionic liquid $\left(\mathrm{BMImBF}_{4}\right)$ increases the diffusivity between two and three orders of magnitude and thus the permeability is enhanced between one and two orders given that the solubility slightly decreases, as can be observed in Table 4 .

Table 4: Diffusivity, solubility and permeability of propylene and propane in HFP-PVDF and PVDF-HFP/BMImBF 4 at $298 \mathrm{~K}$.

\begin{tabular}{llrrr}
\hline & Gas & $\begin{array}{r}\text { D } \\
\left(\times 10^{9} \mathbf{c m}^{2} / \mathbf{s}\right)\end{array}$ & $\begin{array}{r}\mathbf{S} \\
\left(\times 10^{5}\right.\end{array}$ & $\begin{array}{r}\left.\mathbf{P o l} / \mathbf{b a r} / \mathbf{c m}^{3}\right) \\
(\text { barrer })\end{array}$ \\
\hline PVDF-HFP & Propylene & 0.13 & 9.24 & 0.035 \\
& Propane & 0.04 & 7.78 & 0.010 \\
PVDF-HFP/ & Propylene & 37.5 & 1.63 & 1.821 \\
$\mathrm{BMImBF}_{4}$ & Propane & 20.3 & 1.25 & 0.759 \\
$(80 / 20)$ & & & & \\
\hline
\end{tabular}

This increase in diffusivity is a result of the presence of the ionic liquid that is entrapped in the polymer matrix, lowering the rigidity of the polymer chains and increasing the free volume, which facilitates the diffusion of the permeant species.

\section{Conclusions}

Experimental diffusivity, solubility and permeability values of propane and propylene in PVDF-HFP and PVDF-HFP-BMImBF 4 (80/20 wt \%) membranes at different temperatures have been successfully obtained using the time-lag technique. These parameters along with other previously obtained and $\mathrm{C}_{3} \mathrm{H}_{6} / \mathrm{C}_{3} \mathrm{H}_{8}$ separation experimental data will allow the development of a mathematical model able to describe the behavior of the facilitated transport mechanisms of propylene through the composite membrane considering all three mechanisms and design, sizing and optimization of industrial units.

Funding: This research was supported by the Spanish Ministry under the projects CTQ2012-31639 (MINECO, SPAIN-FEDER 2007-2013) and (CTM2013-44081-R).

\section{References}

1. RBN Energy [Internet]. On-purpose propylene via propane dehydrogenation. RBN Energy LLC. [Houston] [Cited 2015 December 21]. Available at: https://rbnenergy.com 
2. Eldridge RB. Olefin/paraffin separation technology: a review. Ind Eng Chem Res 1993;32:2208-12.

3. Baker RW. Research needs in the membrane separation industry: looking back, looking forward. J Memb Sci 2010;362:134-6.

4. Faiz R, Li K. Olefin/paraffin separation using membrane based facilitated transport/chemical absorption techniques. Chem Eng Sci 2012;73:261-84.

5. Das M, Koros WJ. Performance of 6FDA-6FpDA polyimide for propylene/propane separations. J Memb Sci 2010;365:399-408.

6. Fallanza M, Ortiz A, Gorri D, Ortiz I. Polymer-ionic liquid composite membranes for propane/propylene separation by facilitated transport. J Memb Sci 2013;444:164-72.

7. Noble RD. Generalized microscopic mechanism of facilitated transport in fixed site carrier membranes. J Memb Sci 1992;75:121-9.

8. Merkel TC, Blanc R, Ciobanu I, Firat B, Suwarlim A, Zeid J. Silver salt facilitated transport membranes for olefin/paraffin separations: carrier instability and a novel regeneration method. J Memb Sci 2013;447:177-89.
9. Ortiz A, Ruiz A, Gorri D, Ortiz I. Room temperature ionic liquid with silver salt as efficient reaction media for propylene/ propane separation: absorption equilibrium. Sep Purif Technol 2008;63:311-18.

10. Ortiz A, Galán Sanchez LM, Gorri D, De Haan AB, Ortiz I. Reactive ionic liquid media for the separation of propylene/propane gaseous mixtures. Ind Eng Chem Res 2010;49:7227-33.

11. Fallanza M, Ortiz A, Gorri D, Ortiz I. Experimental study of the separation of propane/propylene mixtures by supported ionic liquid membranes containing $\mathrm{Ag}^{+}$-RTILs as carrier. Sep Purif Technol 2012;97:83-9.

12. Ortiz A, Galán LM, Gorri D, De Haan AB, Ortiz I. Kinetics of reactive absorption of propylene in $\mathrm{RTIL}-\mathrm{Ag}^{+}$media. Sep Purif Technol 2010;73:106-13.

13. Ortiz A, Gorri D, Irabien T, Ortiz I. Separation of propylene/ propane mixtures using $\mathrm{Ag}^{+}$-RTIL solutions. Evaluation and comparison of the performance of gas-liquid contactors. J Memb Sci 2010;360:130-41.

14. Yampolskii Y, Pinnau I, Freeman B. Material science of membranes for gas and vapor separation. John Wiley: Chippenham, 2006. 